REFRACTOMETRIC EXAMINATION OF OILS AND FATS.

\title{
XXXII.-A Simple Thermostat for Use in Connection with the Refractometric Examination of Oils and Fats.
}

By Thomas Edward Thorpe, C.B., F.R.S.

In the Government Laboratory, as in other laboratories where large numbers of commercial oils and fats, and especially butter, are required to be examined, the Zeiss butyro-refractometer has proved to be of great service. As is well known, it is generally necessary in using the instrument to allow a current of water of constant temperature to flow through the apparatus for some little time before making the readings, and it was with the view of rapidly and easily obtaining this current that the little contrivance seen in the accompanying figure was devised in substitution for the special heating arrangement supplied with the instrument, which was found to be somewhat cumbrous and uncertain in action.

The temperature employed in the refractometric examination of butter-fat is $45^{\circ}$, and it must be understood, therefore, that the details of the arrangement as described have been adjusted in order that the circulating water shall give this temperature as indicated by the thermometer attached to the refractometer. It is, however, possible, by a slight rearrangement of the details, to obtain a considerable range of constant temperatures.

The principle of the thermostat and the method of working it will be obvious from the figure. The apparatus consists essentially of a vessel for generating steam or other appropriate vapour, containing a coil through which the current of water flows and is heated before it passes through the refractometer or other instrument, the position of which is shown in the drawing at $Z$, by which the observations at a given constant temperature are to be made.

In the figure (p. 258), A represents a metallic cylindrical vessel $5 \mathrm{~cm}$. in diameter and $5 \mathrm{~cm}$. deep, containing approximately 100 c.c. of water, \&c., for generating steam, \&c.

$B$, a conical steam-chamber, in which the coil, C, is suspended and heated by the steam from A. The diameter of cover is $10 \mathrm{~cm}$.

C, a coil of copper or "compo," tubing, from $25 \mathrm{~cm}$. to $30 \mathrm{~cm}$. long, and having an internal diameter of about $3 \mathrm{~mm}$. The current of water passing through the coil is heated before entering the instrument, Z.

$D$, a reflex condenser, preferably of glass, held in position by a tubulure, $d$, provided with a sliding or screw cap to prevent loss of the liquid in $\mathbf{A}$.

E, an arrangement for maintaining a constant head of water at the 
point of entrance to the coil, so as to reduce variations in the temperature of $\mathrm{Z}$, due to fluctuations in the pressure of the current of water. It consists of an outer metal tube, through which a narrow central tube rises, and serves as an overflow when the water in the outer tube has reached the level of its upper orifice.

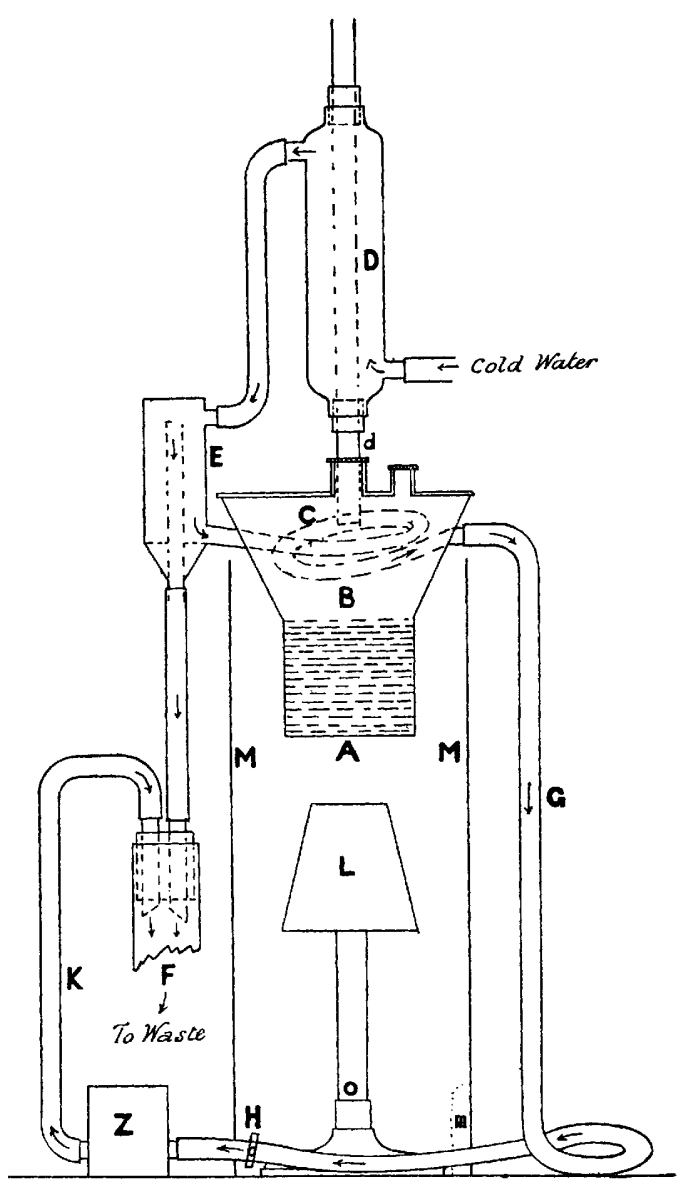

F, a stout glass tube, acting as a gauge or "tell-tale," to indicate whether or not the overflow and the current of water through the instrument, $Z$, are running properly.

G, a thick-walled caoutchouc tubing (for example, pressure-tubing), to minimise loss of heat during the passage of water from the coil to the instrument, $Z$. 
ACTION OF NITROGEN SULPHIDE ON ORGANIC SUBSTANCES. 259

H, a screw clamp, for regulating the rate of flow of water through the coil and through the instrument, $Z$. The final adjustment of the desired temperature may be made by means of the screw clamp.

$K$, a caoutchouc tubing, carrying away waste water from the instrument, $\mathrm{Z}$, and terminating in the gauge, $\mathrm{F}$, where the rate of the current can be observed.

L, Bunsen burner with cone.

M, a metallic cylinder for protecting Bunsen flame from draughts, and to reduce loss of heat due to radiation from walls of the steam generator. At the foot is a semicircular opening, $m$, for the admission of the gas tube.

This little apparatus has been in use for some years in the Government Iaboratory, and has been found to answer its purpose sufficiently well. Only a few minutes are needed to bring it into action, and, under ordinary working conditions, it is readily possible to secure a steady current of water, not varying more than $0 \cdot l^{\circ}$ or so in temperature throughout the day.

The Government Laboratory, LONDON. 